\title{
Implementing High Performance Voltammetry Simulation using the Implicit Parallel Algorithm
}

\author{
Shaltiel Eloul, Richard G Compton* \\ ${ }^{*}$ Corresponding Author, Department of Chemistry, Physical and Theoretical Chemistry, Oxford \\ University, South Parks Road, Oxford OX1 3QZ, United Kingdom. Email: \\ richard.compton@chem.ox.ac.uk
}

\begin{abstract}
The numerical simulation of voltammetry and amperometry at a microdisc electrode is implemented with a parallel algorithm using GPU (CUDA in $\mathrm{C}++$ ) and adapting the implicit finite difference approximation with the ADI method. This leads to high performance simulation of the current response whilst maintaining the high standard of accuracy required for such electrochemical systems. We gained a maximum speed-up of 20 times with a standard graphic card specifications (Quadro K4000) in comparison to a single core of $3.2 \mathrm{Ghz} \mathrm{CPU}$. The simplified allocation and data flow allows an accessible code which produces a starting point for researchers to manipulate the code to study new problems in electrochemical and surface reaction systems that require high performance simulation.
\end{abstract}

Keywords: Voltammetry, GPU, simulation, microdisc electrode simulation, Finite Difference, CUDA, Adsorption

\section{Introduction}

The numerical modelling of the current-time response from electrodes in physicochemical systems is key to understanding mass transport and kinetics in electrochemical cells. [1-3]. One of the strongest tools is voltammetry, where a scanning potential applied to an electrode allows the investigation of surface kinetic processes and determination of the diffusion coefficient of electroactive species. In another important technique, amperometry, the investigation of mass transport and especially diffusion can be achieved by holding the electrode at a fixed potential to measure current over time from the oxidation or reduction of adsorbing or reacting entities.

Experimental electrochemical systems are known to be accurate and are widely used as sensitive and quantitative analytical methods, and with conjunction of ac- 
curate simulation, physical parameters can be extracted. These include diffusion coefficients, reaction mechanisms, kinetic constants, adsorption and catalytic parameters $[4,5]$. Finite Difference algorithms are widely used for the latter purpose, especially among researchers, due to the easy manipulation of the code to get a deep understanding of subtle effects on the transport, but in general, it involves the modelling of relatively simple geometries.

Performance and accuracy are a significant part of the modelling methods, and uncompromising space grids or time steps [1,6], can make calculations be very long, even for relatively simple routine investigations for example, extraction of kinetic parameters and reaction mechanism with a non-linear boundary condition problem $[3,7]$ or studying the life cycle of thousand charge/discharge processes in a multi-dimensional battery cell $[8,9]$. Therefore, high performance simulation is important for making the problems practicable for study and application.

Recently, the simulation of voltammetry using the graphic processor unit (GPU) was introduced for the solution of the finite difference problem via the explicit method [10]. Although the explicit method is a natural parallel problem, it is numerically unstable in comparison for the implicit method as shown for voltammetry problems by Bieniasz et al [11]. Thus, finite difference studies generally use the implicit method to avoid inaccuracy, and sometimes utilize the interface OpenMP (Open Multi-Processing) or the high performance implementation of MPI (Message Passing Interface) to gain a fair performance [6]. However, with growing interest in parallel algorithms using GPU, very recent computational studies show that changing the naturally sequential Thomas algorithm to solve the dominant diagonal matrix with the a less efficient but inherently parallel algorithm 'Parallel Cyclic Reduction' (PCR), enables high performance in recent GPU architectures [12-14]. GPU implementations were reported for heat transfer [13] and are popular for fluid dynamics calculations [15], however, they have not yet introduced for electrochemical and surface kinetics either for research or applications.

We here introduce the Finite Difference simulation using the GPU for the implicit case of voltammetry at a disc electrode, using a two dimensional grid and kinetic boundary conditions for charge transfer in the case of redox reaction between species with equal diffusion coefficients. We also provide a simulation for the case of amperometry. The code provides a starting point for many other modelling systems that can be achieved by modifying and altering the code, such as complex simulation of multi-species with unequal diffusion coefficients, multi-reaction mechanism, adsorption problems and non-linear boundary conditions, or arrays. The code itself is written in an accessible way using CUDA with $\mathrm{C}++$. That said, we optimize the code to be easy to understand rather than to be fully performance optimized. The 
results were also tested in comparison to the serial code with the Thomas algorithm and the analytical theoretical values for voltammetry.

\section{Physical model}

We model a representative electrochemical system of statistical diffusion towards a disc electrode. This case is generally assumes a full supporting electrolyte, where migration is negligible[16]. On the elecrode surface, we use a kinetic and thermodynamic boundary condition with the widely used Butler-Volmer expression for the reaction mechanism on the disc surface[5].

The geometry is illustrated in figure 1a. Using axial symmetry around $r=0$ (figure 1b), we can solve Fick's second law for a cylindrical space [17]:

$$
\frac{\partial c}{\partial t}=D\left(\frac{\partial^{2} c}{\partial r^{2}}+\frac{\partial^{2} c}{\partial z^{2}}+\frac{1}{r} \frac{\partial c}{\partial r}\right)
$$

where $r$ and $z$ are the cylindrical coordinates, as shown in figure 1b. $D$ is the diffusion coefficient, $c$ is the concentration of the active species in an electrochemical cell with the redox couple (A and $\mathrm{B})$. We define two distances in the $r$ direction, the disc electrode radius $\left(r_{d}\right)$ and the surface radius $\left(r_{s}\right)$ as illustrated in figure 1.

$$
D(X)=\frac{6 X^{2}+2 R_{p} X}{6 X^{2}+9 R_{p} X+2 R_{p}^{2}} \cdot D_{0}
$$

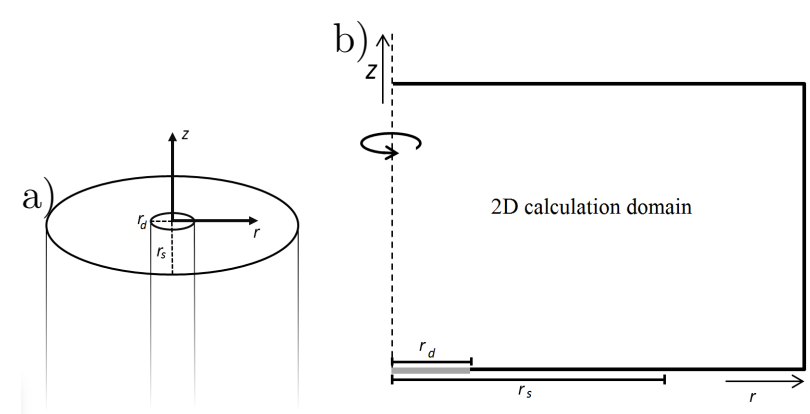

Figure 1: 3D Illustration of the microdisc electrode surrounded by an insulator (a) and the 2D system in the simulation model (b).

The electron transfer electrochemical reaction on the electrode $r \leq r_{d}$ :

$$
A \leftrightarrows B+e^{-}
$$


The kinetics of the reaction on the electrode is defined by the boundary condition as shown below. Assuming equal diffusion coefficients for both species, A and B, and a single step reaction of A to B, we can solve Eq.1 only for species A, and determine the concentration of species B by:

$$
1=\frac{[B]+[A]}{[B]^{*}+[A]^{*}}
$$

where $[\mathrm{A}]^{*}$ is the initial concentration of species $\mathrm{A}$, and $[\mathrm{B}]^{*}$ is the initial concentration of $\mathrm{B}$.

In the work reported below, $[B]^{*}$ is essumed to be zero.

\subsection{Boundary Conditions}

In voltammetry, the scanned potential $(\mathrm{E} / \mathrm{V})$ is applied on the electrode with a scan rate $\nu(V / s)$. At $z=0$ we define the kinetics at the adsorbing surface $\left(r_{d}<\right.$ $\left.r \leq r_{s}\right)$, and the electrode boundary condition $\left(r \leq r_{d}\right)$. At the disc electrode, the letter is set to be the flux of each species related to the electrode potential $(\mathrm{E} / \mathrm{V})$ according to the Butler-Volmer equation [5]:

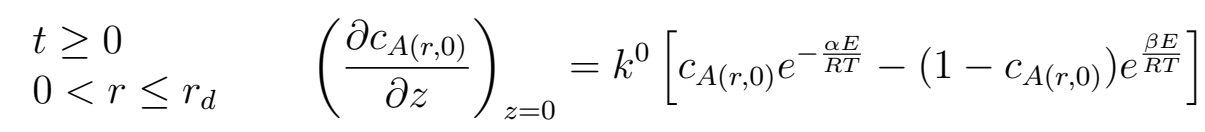

$F$ is the Faraday constant, and $R$ and $T$ are the gas constant and temperature, respectively. $k^{0}$ is the kinetic constant, and $\alpha$ and $\beta$ are the transfer coefficients for the reaction.

In the case of voltammetry, $E$ depends on the potential scan rate $(\nu)$ which is applied on the electrode via:

$$
\begin{array}{rll}
\text { Forwardscan } & E_{\min } \rightarrow \phi_{\max } & E(t)=E \nu+E_{\min } \\
\text { Backwardscan } & E_{\max } \rightarrow \phi_{\min } & E(t)=E_{\max }-E \nu
\end{array}
$$

$E_{\text {min,max }}$ are the lowest negative and highest positive potentials in the (cyclic) voltammetry, respectively. The maximum time $\left(t_{\max }\right)$ is chosen to be a full time of one cycle in the cyclic voltammetry:

$$
t_{\max }=\frac{2\left(E_{\max }-E_{\min }\right)}{\nu}
$$

In the case of amperometry, we adjust the potential to a constant high potential where no kinetic barrier exists and the current is then driven only by diffusion. In 
the case of high potential, very small time steps should be included due to the large gradients in concentration adjacent to the electrode. However, since the long time steady state behaviour is of interest we must use an expanding time grid to solve the problem for short and long time, bearing in mind that this can be a source of instability [11] and thus the expanding factor should be adjusted carefully.

On the other boundaries of the cell, we define a wall/insulation boundary condition for $\mathrm{A}$ and $\mathrm{B}$ :

$$
\begin{aligned}
& t \geq 0 \\
& r_{d}<r, z=0 \quad\left(\frac{\partial[A]}{\partial z}\right)=\left(\frac{\partial[B]}{\partial z}\right)=0
\end{aligned}
$$

where $r_{\max }$ and $z_{\max }$ are set to be the cell boundaries which is generally far enough to affect diffusion in the time scale of experiment. Invariably, we chose $r_{\max }$ and $z_{\max }$ to be larger size than $6 \sqrt{D t_{\max }}$ in both directions $(r, s)$, to simulate an "infinite cell" $[5]$.

From symmetry, the flux across the $z$ axes can be set to zero:

$$
\left(\frac{\partial[A]}{\partial z}\right)_{r=0}=\left(\frac{\partial[B]}{\partial z}\right)_{r=0}=0
$$

Finally, the edges in the outer space of the simulation set to the bulk concentration of species A:

$$
c_{r_{\max }}=[A]^{*}, \quad c_{z_{\max }}=[A]^{*}
$$

\subsection{Current Calculation}

The flux inward the disk electrode is given by:

$$
0<r \leq r_{d} j=\left(\frac{\partial[A]}{\partial z}\right)_{Z=0}
$$

Integrating the flux $(j)$ over the disc, we obtain the net flux $(J)$ inward the disc electrode and thus the Faradaic current of the one electron redox reaction is:

$$
I=2 \pi F[A]^{*} D \int_{0}^{r_{d}} j_{r, 0, t} r \mathrm{~d} r
$$

\section{Numerical Methods}

The partial differential equation is discretized over a spatially expanding $2 \mathrm{D}$ grid with $m_{\max }$ rows and $n_{\max }$ columns $\left(r\left(n_{\max }\right) \times z\left(m_{\max }\right)\right)$ which also gives the matrix 
sent for calculation. This space is meshed generally with an expanding grid around the nodes: $r_{0}, r_{d}, r_{\max }, r_{z}$, where a large perturbation of flux and discontinuities are expected and high accuracy is needed to get a converged result. This can be seen in figure 2a which shows the grid points next to the edge of the electrode and the supporting insulator. The first differences around the nodes have minimum $\delta r$ and $\delta z$ values, after which the differences grow exponentially in space $(\mathrm{r}, \mathrm{z})$, before being compressed again around the boundaries $r=0$, and $r_{\text {max }}$. The concentration equations is set for each species (in this case only for species A) in the space.

a)

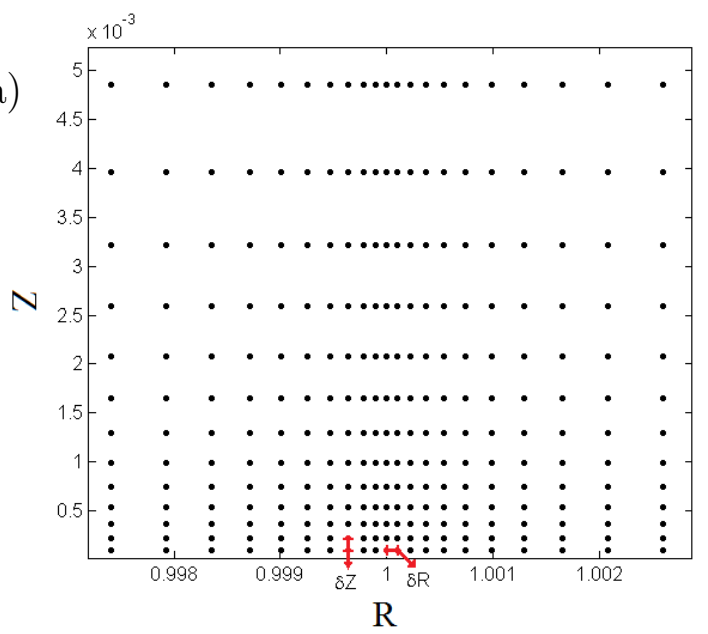

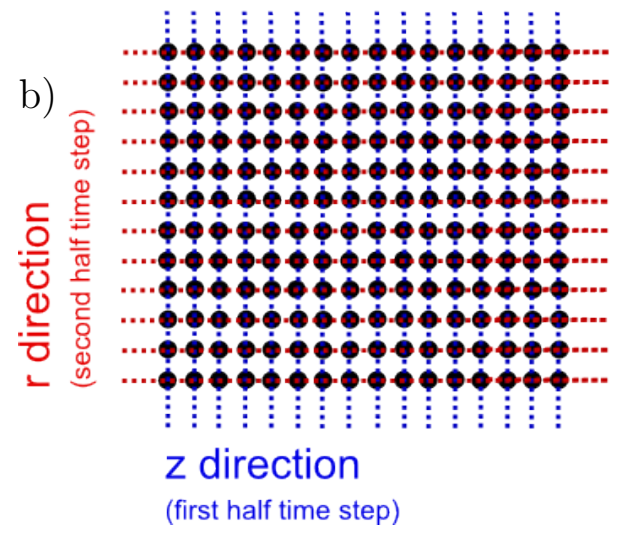

Figure 2: (a) The expending grid next to the nodes. (b) Illustration of the ADI method algorithm.

The partial differential equations for both species, along with the boundary conditions are solved numerically with the finite difference method for each time step, using the implicit Crank-Nicolson method [18]. The alternating direction implicit (ADI) method is used to approximate the implicit solution $[6,13,19]$. Within each time step the calculation is divided into two half time steps where at the first half step the $z$ direction is defined implicitly and the $r$ direction is defined explicitly and vice versa for the second half step [19]. The ADI method allows the solution of each column $(\mathrm{j})$ in the $z$ direction or row (i) in the $r$ direction to be solved in parallel. At each row or column there are a set of equations that form tri-diagonal matrices that need to be solved. In the algorithm the discretization of the set of equations is in the form:

$$
\alpha_{i, j} c_{i, j-1}+\beta_{i, j} c_{i, j}+\gamma_{i, j} c_{i, j+1}=\delta_{i, j}
$$


for the $z$ direction from $\mathrm{j}=0$ to $\mathrm{j}=\mathrm{m}$, or

$$
\alpha_{i, j} c_{i-1, j}+\beta_{i, j} c_{i, j}+\gamma_{i, j} c_{i+1, j}=\delta_{i, j}
$$

for the $r$ direction from $\mathrm{i}=0$ to $\mathrm{i}=\mathrm{n}$. ( All coefficients for the domains and boundaries are shown in the code or in the literature [6]). The coefficients $\alpha, \beta, \gamma$ form a tridiagonal dominant matrix (A) which satisfies the equation:

$$
A^{-1} b=c
$$

The normal use of the Thomas algorithm for Gauss elimination in the case of a diagonal dominant matrix is carried out with linear efficiency $(O(n))$. OpenMP or MPI is a good way to increase the parallelism and create a better performance, optimizing several cores, when each core solves a full row or column in the concentration matrix. However, implementing the algorithm on GPU (to have many threads) makes the algorithm memory usually limited. Therefore, changing the algorithm to a less efficient but inherently parallel algorithm overcomes the memory traffic limit and a high performance calculation can be achieved. Parallel Cyclic Reduction is an inherently parallel algorithm and used to solve the diagonal dominant matrix in many previous finite difference models. Its efficiency is linear-logarithmic, $O(n \log n)$, but can be easily spread to many threads in the current GPU architectures. We therefore use the PCR algorithm to solve the set of concentration equations of each column in the $z$-direction and row in the $r$-direction. The pseudo code and details on the PCR algorithm can be found in the literature $[12,14]$.

\subsection{Memory allocation in the GPU device}

In order to make the algorithm performance optimized to GPU processing, care needs to be take when assigning arrays to the global or shared memory of every block. In some cases, in order to get better performance on the GPU it is necessary to divide the matrix to smaller matrices that can be fit the 'cache' level, to fully utilize the traffic between the various cache levels and the wraps or blocks. This for example can be achieved by the technique of dividing the matrix into smaller sub matrices that can efficiently calculated with the PCR algorithm and use the Thomas algorithm to connect back the sub-matrices into the origin matrix [14]. For our purpose, we use a simplified memory allocation (discussed below) in order to make the shared code accessible as a starting point for high performance voltammetry calculations, so a scientist can relatively easy take the CUDA code, and alter the shared code further for their specific study. The allocation of the main pseudo 2D array of initial concentration values of size equals $m \times n$ is created on the host and is 
allocated in the global memory of the device along with the constant 1D array values of the expanding $\mathrm{z}$ grid $\left(z_{0}-z_{m}\right)$, and the constant $1 \mathrm{D}$ arrays along the $r$ direction $\left(r_{0}-r_{n}\right)$.

These arrays along with constant parameters, and atomic variable for integration summation, are used in the kernels, and the coefficients arrays are defined in the kernel with shared memory for each block of threads. We use the allocation where each row or column is assigned to one block of threads. The mapping into the block is illustrated in figure 3. This limits the size of a direction to the maximum size of blocks in the specific GPU architecture, but it is enough for most practical applications whilst keeping the coding fairly simple. In order to make larger grids, it is necessary to modify the mapping strategy with extra coding $[12,14]$.

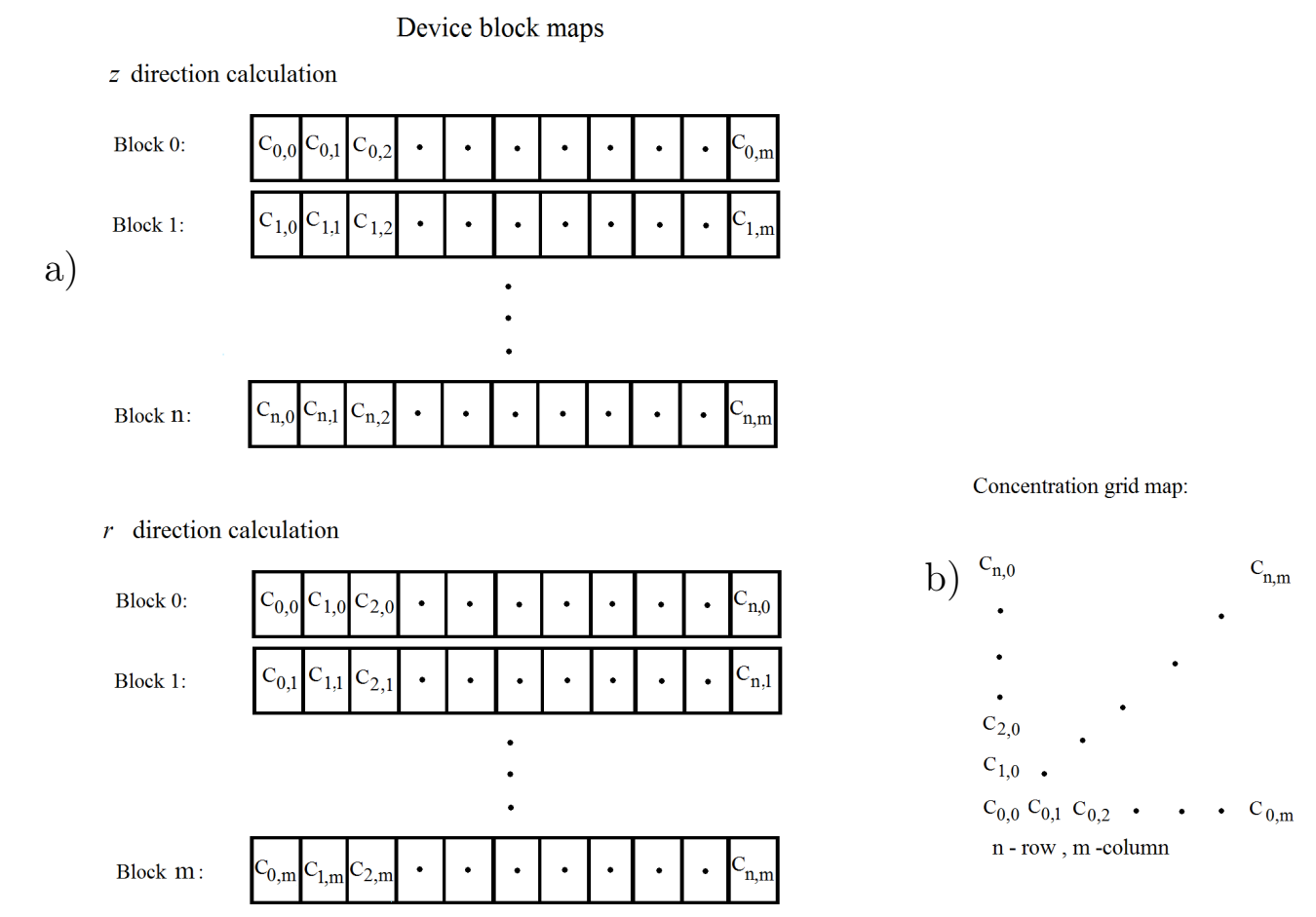

Figure 3: Illustration of allocation in the blocks (a), where each block is assigned to a row or a column in the concentration matrix (b).

\subsection{Implementation}

The parallelization of ADI solver using NVIDIA CUDA was implemented through $\mathrm{C}++$. The test machine was a Xeon E5-1650, 3.2GHz. The GPU tested is a NVIDIA 
Quadro K4000 with 4GB graphics memory and a 48KB shared memory (Kepler architecture), with 768 cores.

We use the serial solving method where each direction is solved in a different kernel. Optimization for a better performance was found using the transpose of the array matrix between each direction, and using texture memory, but we can avoid this alteration in this stage [12]. The diagram in figure 4 shows the implementation on the GPU device. The calculation method is divided into three serial kernels to get synchronization between the blocks. The first is for solving the $z$ direction for each half time step by assigning the coefficients in the matrix including the respective boundary conditions, and using the PCR algorithm it returns the new concentration maps. The second kernel makes the integration using atomic addition to get global reduction and copy back the concentration to use in the third kernel. In the same kernel, we solve the $\mathrm{r}$ direction, and the third kernel is for saving results and parameter updates. It is noted that the $z$ and $r$ calculation kernels re-assign $\alpha, \beta$, and $\gamma$ coefficients at each step which is not essential in this calculation, but in some cases it is needed as in the case of bulk or surface reaction and adsorption, or when adding a drift (migration) term to the diffusion equation [6].

\section{Calculations}

In the following, we show typical current responses of voltammetry and amperometry simulations carried out on the GPU with the PCR algorithm. First, we show the validation of a voltammetry without kinetic limitation to validate the result with theory, and discuss the performance in comparison to the simulation carried out with the Thomas algorithm on the GPU. Second, we test the simulation in various electrode kinetic constants voltammetry and the case for amperometry, in order to demonstrate the ability to model the common use of electrochemical simulations. Third, we discuss the performance of the GPU calculation in comparison to calculations on the CPU at various matrix sizes.

It is convenient to convert the calculation and output results to dimensionless units where $\phi=E F / R T$ is a dimensionless potential $\tau=D t / r_{d}^{2}$ and therefore the scan rate is defined as: $\sigma=\phi \cdot r_{d}^{2} / D[17]$. The dimensionless flux is defined as : $J=$ $I(A) / F D[A]^{*} r_{d}$, and lastly, the kinetic constant $k^{0}(\mathrm{~m} / \mathrm{s})$ is defined in dimensionless units as $\mathrm{K}=k^{0} r_{d} / D$, simplifying all calculations are presented in dimensionless form.

\subsection{Typical Voltammetry}

The results of GPU calculations for typical voltammetry is shown in figure 5 for two dimensionless scan rates. This shows the net (dimensionless) flux response as 
kernel 1:

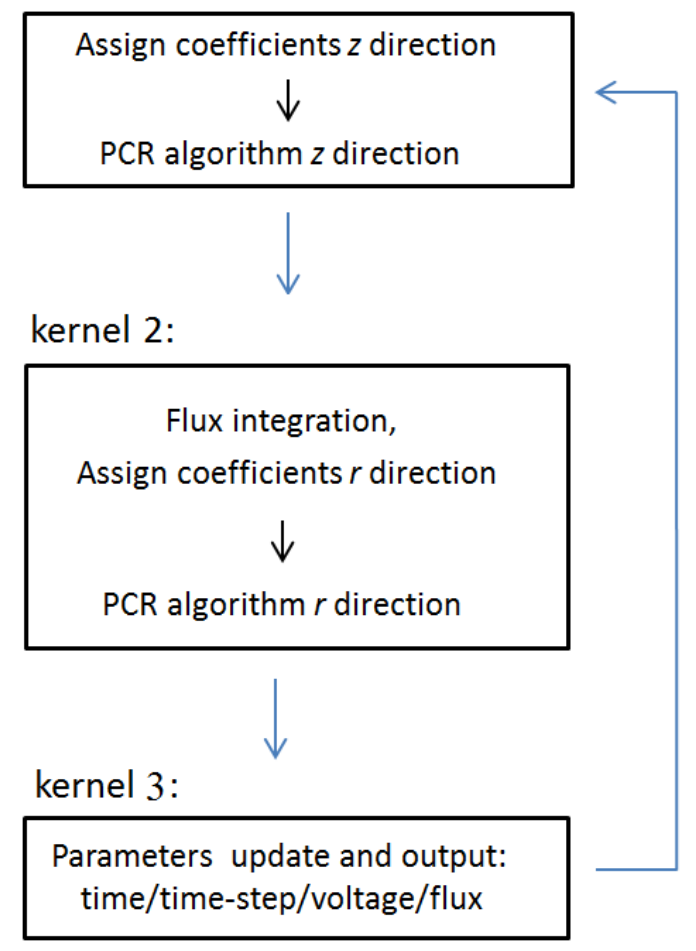

Figure 4: Illustration of the data flow at each time step.

function of (dimensionless) potential applied on the electrode surface when there is no kinetic limitation on the electrode reaction (reversible process).

The results in figure 5 show high accuracy and can be validated and approximated with theoretical values of the peak values by the formula[5]:

$$
J_{\text {peak }}=-4 r_{d}[A]^{*}(0.34 \exp (-0.66 \sqrt{\sigma})+0.66-0.13 \exp (-11 / \sqrt{\sigma})+0.351 \sqrt{\sigma})
$$

The results of the simulation show a deviation up to $0.02 \%$. The simulations were also calculated in the CPU using the traditional method of Thomas algorithm and show similar results, with a deviation of a maximum $2 \cdot 10^{-8}$ between the net fluxes. This is a negligible error. However it is a crucial observation to validate the model due to the differences between the methods for eliminating the matrix. note the PCR algorithm has a significantly larger amount of arithmetic operations per time step, that might express more rounding errors from the approximations made in the model. In terms of performance, the calculation of voltammetry in the CPU 


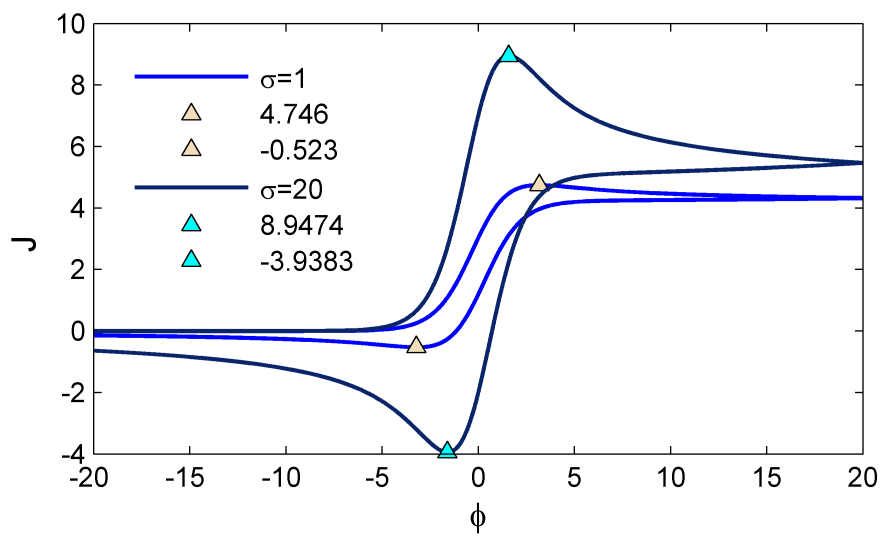

Figure 5: Calculation of a characteristic voltammogram for two dimensionless scan rates, $\sigma=20$, and $\sigma=1$.

with the Thomas algorithm took 22 minutes in comparison with 72s on the GPU, a speed up factor of 19 for this hardware configuration.

\subsection{Kinetics in voltammetry, and the simulation of amperometry}

The results for voltammetry calculated on the GPU in the case of various kinetic dominant constants $(K=1000,100,10)$ are shown in figure 6 a and for the amperometry simulation applying a high potential to measure the decay of current, is presented in figure $6 \mathrm{~b}$. The results are in full agreement with the simulation made on the CPU [20]. The maximum difference between the calculations found to be bound to $5 \cdot 10^{-8}$. It is important to note that when using an expanding time step, for achieving the steady state behaviour at very long time, we found that the PCR algorithm is more sensitive to numerical oscillation than the Thomas algorithm for this model of amperometry due to accumulated error when using the expanding time step. This is reasonable since many more arithmetic operations are made in the PCR algorithm which will thus grow the error faster. 

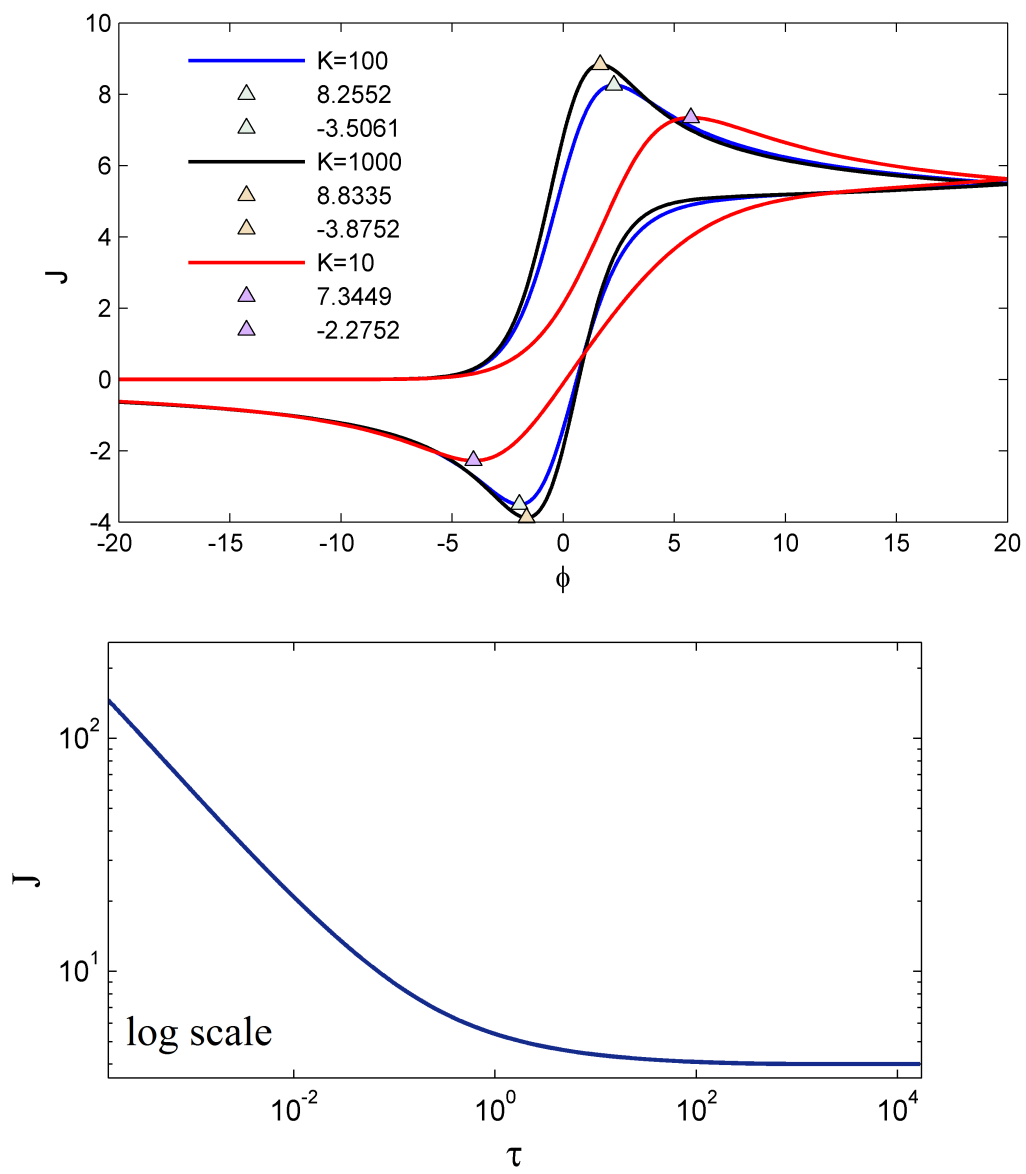

Figure 6: a) Calculation of a characteristic voltammogram for a various dimensionless kinetic constants. b) Amperometry simulation plotted on a logarithmic scale. 


\subsection{Performance tests}

We evaluate the performance of the GPU implementation relative to the CPU for various concentration matrix sizes in Table 4.3. It is shown that a significant increase of speed is gained in small and large size matrices. The result for maximum speed up at a matrix size of 103X103 is specific to the GPU architecture used here (Kepler architecture and 768 cores). The different speed ups for various matrix sizes and the maximum speed up at 103X103 are due to better time optimization between the memory traffic of the various cache memory levels to the computing time of the threads for this specific GPU. This keeps the maximum number of cores busy during the calculations. The NVIDIA profiler analysis implies that increasing the number of cores would increase significantly the speed and therefore it is important to mention that the algorithm made on only single core $\mathrm{CPU}$ rather than using parallel CPU implementation, since both the number of cores in CPU often varies , and also the number of GPU cores tested is relatively low compared to other models. Bearing in mind that the serial Thomas algorithm is traffic limited, we found that using the interface OpenMP (Open Multi-Processing) for eight cores in the ADI method increases by a factor of three the speed on average. Running the program in the NVIDIA visual profiler implies that most of the time is spent on arithmetic calculations which requires the assigning of the coefficients before the PCR algorithm. Therefore, higher performance can be achieved by pre-assigning parameters in the shared memory and/or using the texture memory.

\begin{tabular}{lll}
\hline Matrix size & Speed up GPU/CPU & maximum difference in flux \\
\hline 32 X32 & 8.2 & $6.48 \times 10^{-8}$ \\
45X45 & 13.3 & $1.08 \times 10^{-9}$ \\
103X103 & 20.3 & $1.2 \times 10^{-8}$ \\
400X400 & 12.09 & $2.0 \times 10^{-7}$ \\
732X732 & 11.5 & $3.0 \times 10^{-8}$ \\
1016X1016 & 14.5 & $8.0 \times 10^{-8}$ \\
\hline
\end{tabular}

Table 1: Performance evaluation for a specific CPU 1core Xeon E5-1650, 3.2Ghz, and GPU device with Kepler architecture and 768 cores.

\subsection{Program availability}

The program is provided by request from the author or can be downloaded at http://compton.chem.ox.ac.uk. 


\section{Conclusions}

Simulation code is introduced using CUDA NVIDIA for the parallel computing of voltammetry and amperometry of a small disc in a $2 \mathrm{D}$ cylindrical system with a boundary condition of kinetic charge transfer, and equal diffusion coefficients for both species in a redox reaction. The implementation is used via the implicit finite difference approximation with the ADI method and the PCR algorithm. This introduces high performance simulation of the current response whilst maintaining the high accuracy required in electroanalytical studies of mass transport and surface reaction/adsorption. The simulation was validated and tested in comparison with the Thomas algorithm and theoretical values of the peak currents and steady state values. We gained up to 20 times faster simulation of voltammetry with a relatively standard graphic card (Quadro K4000) in comparison with a single core of Xeon 3.2GHz CPU. The simplified allocation and data flow used allows an accessible code which is a starting point for researchers to manipulate the code via $\mathrm{C}++$ to study new models of electrochemical and surface reaction systems which require high performance, such as kinetic studies and highly costs simulation for many species with unequal diffusion coefficients.

\section{Acknowledgement}

The research leading to these results has received funding from the European Research Council under the European Union's Seventh Framework Programme (FP/20072013) / ERC Grant Agreement n. [320403]. 


\section{References}

[1] D. Britz, Digital Simulation in Electrochemistry, volume 666, Springer, 2005.

[2] H. Angerstein-Kozlowska, J. Klinger, B. Conway, Computer simulation of the kinetic behaviour of surface reactions driven by a linear potential sweep: Part i. model 1-electron reaction with a single adsorbed species, Journal of Electroanalytical Chemistry and Interfacial Electrochemistry 75 (1977) 45-60.

[3] J. A. Alden, R. G. Compton, A general method for electrochemical simulations. 1. formulation of the strategy for two-dimensional simulations, J. Phys. Chem. B 101 (1997) 8941-8954.

[4] A. J. Bard, L. Faulkner, Electrochemical Methods: Fundamentals and Applications, John Wiley and Sons, 2001.

[5] R. G. Compton, C. E. Banks, Understanding Voltammetry, Imperial College Press, 2006.

[6] R. G. Compton, E. Laborda, K. R. Ward, Understanding Voltammetry - Simulation of Electrode Processes, Imperial College Press, 2013.

[7] H. Balslev, D. Britz, Direct digital simulation of the steady-state limiting current at, Acta Chemica Scandinavica 46 (1992) 949-955.

[8] S. Elul, Y. Cohen, D. Aurbach, The influence of geometry in 2d simulation on the charge/discharge processes in li-ion batteries, Journal of Electroanalytical Chemistry 682 (2012) $53-65$.

[9] G. Ning, R. White, B. Popov, A generalized cycle life model of rechargeable li-ion batteries, Electrochimica Acta 51 (2006) 2012-2022. Cited By 109.

[10] I. J. Cutress, R. G. Compton, Using graphics processors to facilitate explicit digital electrochemical simulation: Theory of elliptical disc electrodes, Journal of Electroanalytical Chemistry 643 (2010) 102-109.

[11] L. K. Bieniasz, O. Østerby, D. Britz, Numerical stability of finite difference algorithms for electrochemical kinetic simulations: Matrix stability analysis of the classic explicit, fully implicit and crank-nicolson methods and typical problems involving mixed boundary conditions, Computers \& Chemistry 19 (1995) 121 136. 
[12] Y. Zhang, J. Cohen, J. D. Owens, Fast tridiagonal solvers on the gpu, ACM Sigplan Notices 45 (2010) 127-136.

[13] Z. Wei, B. Jang, Y. Zhang, Y. Jia, Parallelizing alternating direction implicit solver on \{GPUs\}, Procedia Computer Science 18 (2013) 389 - 398. International Conference on Computational Science.

[14] M. Giles, E. László, I. Reguly, J. Appleyard, J. Demouth, Gpu implementation of finite difference solvers, in: Proceedings of the 7th Workshop on High Performance Computational Finance, 2014, pp. 1-8.

[15] N. Sakharnykh, Tridiagonal solvers on the gpu and applications to fluid simulation, in: NVIDIA GPU Technology Conference, 2009.

[16] E. J. Dickinson, J. G. Limon-Petersen, N. V. Rees, R. G. Compton, How much supporting electrolyte is required to make a cyclic voltammetry experiment quantitatively diffusional? a theoretical and experimental investigation, J. Phys. Chem. C 113 (2009) 11157-11171.

[17] J. Heinze, Diffusion processes at finite (micro) disk electrodes solved by digital simulation, Journal of Electroanalytical Chemistry and Interfacial Electrochemistry 124 (1981) 73-86.

[18] J. Crank, P. Nicolson, A practical method for numerical evaluation of solutions of partial differential equations of the heat-conduction type, in: Mathematical Proceedings of the Cambridge Philosophical Society, volume 43, Cambridge Univ Press, pp. 50-67.

[19] I. Lindemuth, J. Killeen, Alternating direction implicit techniques for twodimensional magnetohydrodynamic calculations, Journal of Computational Physics 13 (1973) 181-208.

[20] E. Kätelhön, R. G. Compton, Testing and validating electroanalytical simulations, Analyst 140 (2015) 2592-2598. 